\title{
Table of Modified Bernoulli Polynomials
}

\section{By Gertrude Blanch and Roselyn Siegel}

The polynomials tabulated are $b_{k}(x)$, where $b_{k}(x)$ is related to the Bernoulli polynomial $B_{k}(x)$ by the formula

$$
b_{k}(x)=\left[(-1)^{k} \frac{1}{2}(2 \pi)^{k} B_{k}\left(\frac{x}{2 \pi}\right)\right] / k !
$$

Range of parameters: $k=1$ (1) $11 ; x=\pi y / 36, y=0(1) 36$. The entries are given to 17 decimal places. The functions were computed in connection with the tabulation of a solution to the telegrapher's equation by George E. Forsythe.

\section{Definitions}

The polynomials $b_{k}(x)$ tabulated below can be identified with the Bernoulli polynomials $B_{k}(x)$ by the following relations:

For $_{-}^{-} 0<x \leq \pi$, we have

$$
b_{k}(x)=\frac{(-1)^{k} \frac{1}{2}(2 \pi)^{k} B_{k}\left(\frac{x}{2 \pi}\right)}{k !} .
$$

$$
b_{k}(x)=\sum_{n=1}^{\infty} \frac{\sin \left[n x+\frac{1}{2}(k-1) \pi\right]}{n^{k}}, k \geq 1 .
$$

Explicit expressions for the polynomials tabulated here are as follows:

$$
\left.\begin{array}{l}
b_{1}(x)=-\frac{x}{2}+\frac{\pi}{2} ; \\
b_{2}(x)=\frac{x^{2}}{4}-\frac{\pi x}{2}+\frac{\pi^{2}}{6} ; \\
b_{3}(x)=-\frac{x^{3}}{12}+\frac{\pi x^{2}}{4}-\frac{\pi^{2} x}{6} ; \\
b_{4}(x)=\frac{x^{4}}{48}-\frac{\pi x^{3}}{12}+\frac{\pi^{2} x^{2}}{12}-\frac{\pi^{4}}{90} ; \\
b_{5}(x)=-\frac{x^{5}}{240}+\frac{\pi x^{4}}{48}-\frac{\pi^{2} x^{3}}{36}+\frac{\pi^{4} x}{90} ; \\
b_{6}(x)=\frac{x^{6}}{1,440}-\frac{\pi x^{5}}{240}+\frac{\pi^{2} x^{4}}{144}-\frac{\pi^{4} x^{2}}{180}+\frac{\pi^{6}}{945} ; \\
b_{7}(x)=-\frac{x^{7}}{10,080}+\frac{\pi x^{6}}{1,440}-\frac{\pi^{2} x^{5}}{720}+\frac{\pi^{4} x^{3}}{540}-\frac{\pi^{6} x}{945} ; \\
b_{8}(x)=\frac{x^{8}}{80,640}-\frac{\pi x^{7}}{10,080}+\frac{\pi^{2} x^{6}}{4,320}-\frac{\pi^{4} x^{4}}{2,160}+\frac{\pi^{6} x^{2}}{1,890}-\frac{\pi^{8}}{9,450} ; \\
b_{9}(x)=-\frac{x^{9}}{725,760}+\frac{\pi x^{8}}{80,640}-\frac{\pi^{2} x^{7}}{30,240}+\frac{\pi^{4} x^{5}}{10,800}-\frac{\pi^{6} x^{3}}{5,670}+\frac{\pi^{8} x}{9,450} ; \\
b_{10}(x)=\frac{x^{10}}{7,257,600}-\frac{\pi x^{9}}{725,760}+\frac{\pi^{2} x^{8}}{241,920}-\frac{\pi^{4} x^{6}}{64,800}+\frac{\pi^{6} x^{4}}{22,680}-\frac{\pi^{8} x^{2}}{18,900}+\frac{\pi^{10}}{93,555} ; \\
b_{11}(x)=-\frac{x^{11}}{79,833,600}+\frac{\pi x^{10}}{7,257,600}-\frac{\pi^{2} x^{9}}{2,177,280}+\frac{\pi^{4} x^{7}}{453,600}-\frac{\pi^{6} x^{5}}{113,400}+\frac{\pi^{8} x^{3}}{56,700}-\frac{\pi^{10} x}{93,555} .
\end{array}\right\}
$$

\footnotetext{
${ }_{1}$ See H. T. Davis, Tables of the higher mathematical functions, II, 181 (Principia Press, Inc., Bloomington, Ind., 1935).
} 
The entries correspond to the argument $x=\pi y / 36$, for $y=0(1) 36$. The values are given to 17 decimal places, with an error of less than one unit in the last place.

The above functions were obtained in the process of computing the function $G(x, z)$ required in the solution of the telegrapher's equation by George E. Forsythe. ${ }^{2}$

\section{Method of Computation}

The derivatives with respect to $y$ of $b_{k}(\pi y / 36)$, at $y=0$, were computed with the aid of Glaisher's values ${ }^{3}$ of $\pi^{n}$; and the leading forward differences at $y=0$ were then computed from the well-known formulas for differences in terms of derivatives. Let $f(y)$ be a polynomial of degree $k$, and let $\Delta^{p} f_{0}$ indicate the $p^{\text {th }}$ forward difference of $f(y)$ at $y=0$. Then for integral values of $s$,

$$
f(s)=\sum_{p=0}^{k} C_{p} \Delta^{p} f_{0}
$$

where

$$
{ }_{s} C_{p}=s ! / p !(s-p) ! .
$$

If $\Delta^{p} f_{0}$ is not exact, but has the rounding error $\epsilon_{p}$, then from eq 2 the error in $f(s)$, due to using inexact differences, will be

$$
\rho_{s}=\sum_{p=0}^{k}{ }_{s} C_{p} \boldsymbol{\epsilon}_{p} .
$$

In order to insure an error no greater than $2 \times 10^{-18}$ in $f(s)$, the leading differences were computed to a high enough accuracy to satisfy

$$
\left|k_{s} C_{p} \epsilon_{p}\right|<2 \times 10^{-18}, s=36,
$$

${ }^{2}$ See G. E. Forsythe, J. Research NBS 44, 89 (1950) RP 2059; the polynomials $b_{k}(x)$ are termed $(-1)^{k-1} \sigma_{k-1}(x)$ in this paper.

3 J. W. Glaisher, Numerical values of the first twelve powers of $\pi$, of their reciprocals, and of certain other related quantities, Proc. London Math. Soc. VIII. 140 (1877). and the computed differences were tested by generating the last required value (corresponding to $y=36$ ) from eq 2 and comparing the result with the value of the functions computed from eq 1 . This phase of the work was done with the aid of desk calculators.

The leading differences were then key-punched, carefully tested, and used to generate all remaining values on an IBM tabulator, type 405. The last value obtained in the process agreed with precomputed values to within two units in the eighteenth decimal place, and this guaranteed the accuracy of all intermediate values, since each value was based on previously computed values. To eliminate a possible listing error, the values listed on the preliminary manuscript were differenced on an adding machine. Finally the values were summed in groups of nine or ten, and the sums recorded; this was done on the 405 IBM tabulator, from the punched cards. The galley proofs of the printed table were carefully proofread, and then summed in the same groups of nine or ten; from these sums the precomputed sums were subtracted. A zero total insured the agreement of the galley proofs with the tested preliminary manuscript.

The preparation of leading differences at $y=0$, and the checking of the manuscript were performed by Mrs. H. Arens and Misses F. Gordon, B. Harding, S. Marks, and R. Tishman. The computation of the functions on the IBM tabulator was performed by Everett Rea. 


\begin{tabular}{|c|c|c|c|}
\hline$y$ & & $b_{2}\left(\frac{\pi y}{36}\right.$ & $b_{3}\left(\frac{\pi y}{36}\right)$ \\
\hline 0 & 1. 57079632679489662 & 1. 64493406684822644 & 0.00000000000000000 \\
\hline 1 & 1. 52716309549503838 & 1. $5097600868 \quad 17874 \quad 50$ & -.13762180918235785 \\
\hline 2 & 1. 48352986419518014 & 1. 37839382453485641 & -.26361360632113616 \\
\hline 3 & 1. 43989663289532190 & 1. 25083527999917219 & $-.3783076774 \quad 7472292$ \\
\hline 4 & 1.39626 340159546366 & 1. $1270844532 \quad 1082182$ & $-.48203 \quad 630870150612$ \\
\hline 5 & 1. 35263017029560542 & 1.00714 134416980531 & $-.57513 \quad 17860 \quad 59873 \quad 74$ \\
\hline 6 & 1. $308996938995747 \quad 18$ & 0.89100595287612265 & -.65792639560821378 \\
\hline 7 & 1. 26536370769588894 & .77867827932977386 & -.73075242340491421 \\
\hline 8 & 1. 22173047639603070 & .67015832353075892 & -.79394215550836302 \\
\hline 9 & 1. 17809724509617246 & .565446085479077 .84 & -.84782787797694821 \\
\hline 10 & 1. 13446401379631423 & $.46454 \quad 15651 \quad 7473061$ & -.89274187686905775 \\
\hline 11 & 1. 09083078249645599 & $.3674447626 \quad 1771725$ & -.92901643824307964 \\
\hline 12 & 1. 04719755119659775 & $.27415 \quad 5677808037 \quad 74$ & -.95698384815740186 \\
\hline 13 & 1. 00356431989673951 & .18467431074569209 & -.97697639267041239 \\
\hline 14 & $0.95993 \quad 1088596881 \quad 27$ & .09900066143068030 & -.98932635784049923 \\
\hline 15 & .91629785729702303 & +.01713472986300236 & -.99436602972605037 \\
\hline 16 & .87266462599716479 & -.06092 348395734172 & -.99242769438545378 \\
\hline 17 & $.82903 \quad 139469730655$ & -.1351739800 3035194 & -.98384363787709745 \\
\hline 18 & $.7853981633 \quad 9744831$ & -.20561675835602830 & -.96894614625936938 \\
\hline 19 & .74176493209759007 & -.27225181893437081 & -.94806750559065755 \\
\hline 20 & .69813170079773183 & -.33507916176537946 & -.92154000192934994 \\
\hline 21 & .65449846949787359 & -.39409878684905425 & -.88969592133383454 \\
\hline 22 & .61086523819801535 & -.44931069418539518 & -.85286754986249934 \\
\hline 23 & $.56723 \quad 200689815711$ & -.50071488377440226 & -.81138717357373233 \\
\hline 24 & .52359877559829887 & $-.54831 \quad 135561607548$ & -.76558707852592149 \\
\hline 25 & .47996554429844063 & $-.5921001097 \quad 1041484$ & -.71579955077745480 \\
\hline 26 & .43633231299858239 & $-.63208 \quad 11460 \quad 57420 \quad 34$ & -.66235687638672027 \\
\hline 27 & .39269908169872416 & - 66825446465709199 & -.60559134141210586 \\
\hline 28 & $.34906 \quad 58503 \quad 9886592$ & -.70062006550942978 & -.54583523191199958 \\
\hline 29 & .30543261909900768 & -.72917 794861443371 & -.48342083394478940 \\
\hline 30 & .26179938779914944 & -.75392 811397210378 & -.41868043356886331 \\
\hline 31 & $.21816 \quad 615649929120$ & -. 77487056158244000 & -.35194631684260930 \\
\hline 32 & .17453292519943296 & -.79200 529144544236 & -.28355076982441536 \\
\hline 33 & $.130899693899574 \quad 72$ & -.80533 230356111086 & -.21382607857266948 \\
\hline 34 & $.08726 \quad 646259971648$ & -.81485159792944550 & -.14310452914575963 \\
\hline 35 & .04363323129985824 & -.82056317455044629 & -.07171840760207381 \\
\hline 36 & .00000000000000000 & -.82246703342411322 & .00000000000000000 \\
\hline
\end{tabular}




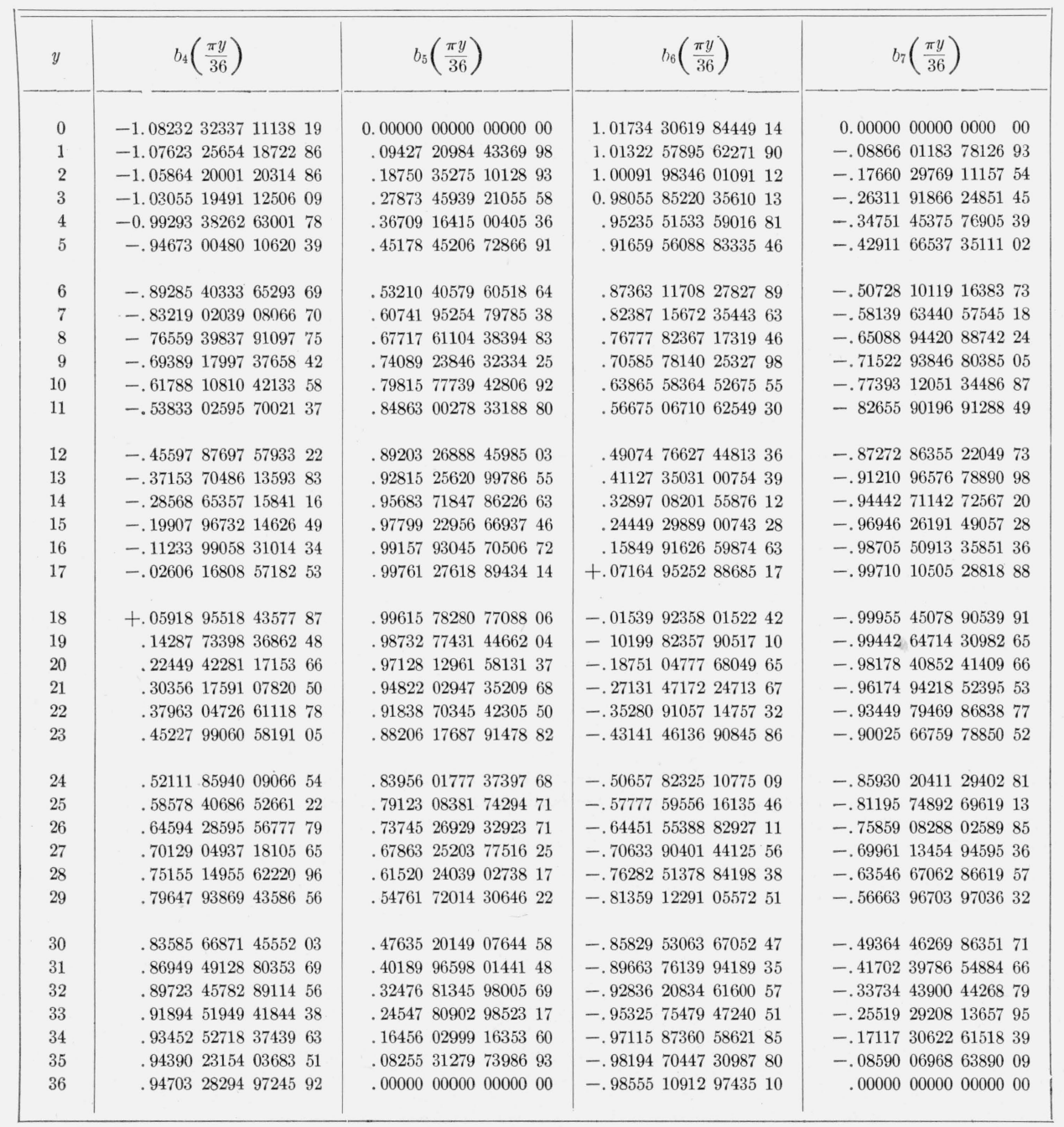




\begin{tabular}{|c|c|c|c|c|}
\hline$y$ & ( & & & $b_{11}\left(\frac{\pi y}{36}\right)$ \\
\hline 0 & -1.00407735619794434 & 0.00000000000000000 & 1.00099457512781809 & 0.00000000000000000 \\
\hline 1 & $-1.0002062153 \quad 5123207$ & $.08750 \quad 96417 \quad 14427 \quad 19$ & 0.99717378965624493 & -.08724208491546146 \\
\hline 2 & -0.98862411833983038 & .17434455345589185 & .98574089377400080 & -.17381817416956597 \\
\hline 3 & $-.96942 \quad 46955 \quad 1758181$ & $.25983 \quad 54624 \quad 82064 \quad 52$ & .96678403074181864 & -.25906740699836828 \\
\hline 4 & $-.9427628715 \quad 46864 \quad 46$ & .34332395094897098 & .94044931415589082 & -.34233915091150030 \\
\hline 5 & -.90885 324570958054 & .42416773839932275 & $.9069396491 \quad 5877037$ & -.42299801247681184 \\
\hline 6 & -.86796791935868985 & $\begin{array}{llll}50174 & 57983 & 79693 & 22\end{array}$ & .86651309955285863 & $-.5004287253 \quad 1720257$ \\
\hline 7 & -.82043352516559570 & .57546326333746770 & .81948081732669662 & -.57404087638346948 \\
\hline 8 & -.76662961113698879 & .64475607664469300 & $.76620 \quad 45548 \quad 99386 \quad 57$ & -.64327343317635518 \\
\hline 9 & -.70698213069305287 & .70909535514539331 & $.707093783780161 \quad 14$ & $-.7075990365 \quad 1383410$ \\
\hline 10 & $-.6419625884 \quad 17234 \quad 17$ & $.76799 \quad 14300 \quad 25604 \quad 20$ & .64260244634120003 & -.76652802564136942 \\
\hline 11 & $-.5720823894 \quad 06 C 7493$ & $.820997538061304 \quad 78$ & $.5732253700 \quad 2507142$ & -.81961216492988551 \\
\hline 12 & $-.4978887384 \quad 6590954$ & .86771313940860151 & $.4994943755 \quad 6645788$ & -.86644804406611699 \\
\hline 13 & -.41996003372151995 & .90778684206293266 & $.4219741127 \quad 1391842$ & -.90668012648152485 \\
\hline 14 & -.33890109752014552 & .94091891692972350 & $.341257658504148 \quad 24$ & -.94000342375893479 \\
\hline 15 & -.25533828583254031 & $.9668633911 \quad 1782576$ & $257961914381331 \quad 71$ & -.96616577687139693 \\
\hline 16 & -.16991451567106943 & .98542971058922323 & .17272283938054904 & -.98496972831754804 \\
\hline 17 & -.08328424836313406 & $996483966673877 \quad 13$ & +.08619055721960066 & -.99627397249515466 \\
\hline 18 & +.00389153516348690 & .99994968418722009 & -.00097562451913894 & -.99999437497382370 \\
\hline 19 & .09095033260849242 & .99580817196968769 & -.08811224487332583 & -.99610455466449792 \\
\hline 20 & $.17723 \quad 30592 \quad 4692769$ & .98409843960279910 & -.17455673160883928 & -.98463602621383768 \\
\hline 21 & .26208889648558269 & .96491668684466608 & -.25965241735906721 & -.96567790325357548 \\
\hline 22 & $.344880020598140 \quad 12$ & .93841537496941997 & -.34275349289009301 & -.93937616638718084 \\
\hline 23 & $.42498 \quad 6183890993 \quad 39$ & $.9048018916 \quad 8990178$ & -.42322986149801534 & -.90593250297857366 \\
\hline 24 & .50180912223335764 & .86433682369105831 & -.50047185966737071 & $-.865602728901174 \quad 44$ \\
\hline 25 & .57477676456699790 & $.817331853002830^{\circ} 49$ & -.57389481044087238 & -.81869480539239803 \\
\hline 26 & .64334722169260079 & .76414729549590576 & -.64294337745762354 & $-.76556 \quad 64670 \quad 2201997$ \\
\hline 27 & $.7070125333 \quad 1151761$ & .70518930169153699 & -.70709 568929680009 & $-.70662 \quad 247950702372$ \\
\hline 28 & $.76530 \quad 2153983308 \quad 55$ & $.6409067418 \quad 37703 \quad 84$ & -.76586720560372143 & $-.64231 \quad 154867604508$ \\
\hline 29 & $.8177861603 \quad 4121956$ & $.57178 \quad 77988 \quad 18210 \quad 97$ & -.81881429846243070 & -.57312290428995489 \\
\hline 30 & .86407816358942493 & .49835629392887837 & -.86553752460058038 & -.49958258464916925 \\
\hline 31 & .90383791298757090 & 42116777187578523 & -.90568456625576022 & -.42224944995177144 \\
\hline 32 & .93677357770985673 & $.3408053725 \quad 2457765$ & -.93895282088460295 & -.34171095419941402 \\
\hline 33 & .96264369614759204 & .25787551795714385 & -.96509162234425446 & -.25857870707130069 \\
\hline 34 & .98125878340587050 & .17300344427249743 & -.98390407870729008 & -. 17348385859250396 \\
\hline 35 & $.99248 \quad 25894 \quad 26702 \quad 14$ & .08682860830249012 & -.99524851447309257 & -.08707234060474899 \\
\hline 36 & .99623300185264790 & .00000000000000000 & -.99903950759827157 & .00000000000000000 \\
\hline
\end{tabular}

Los Angeles, May 6, 1949. 